\title{
In vitro Evaluation of Cobalt-Zinc Ferrite Nanoparticles Coated with DMSA on Human Prostate Cancer Cells
}

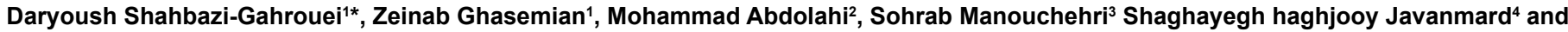 \\ Nasim Dana ${ }^{4}$ \\ ${ }^{1}$ Department of Medical Physics and Medical Engineering, School of Medicine, Isfahan University of Medical Sciences, Isfahan, Iran \\ ${ }^{2}$ Department of Medical Physics and Radiation Technology, School of Paramedical Sciences, Bushehr University of Medical Sciences, Bushehr, Iran \\ ${ }^{3}$ Department of Physics, Malek-Ashtar University of Technology, Shahinshahr, Isfahan, Iran \\ ${ }^{4}$ Physiology research center, Isfahan University of Medical Sciences, Hezar Jerib Street, Isfahan, Iran
}

\begin{abstract}
Toxicity of nanoparticles on the human health is one main feature for successful application of nanoparticles in medicine. In this paper, the cytotoxicity effects of CZF-MNPs and CZF-MNPs @ DMSA were evaluated. For this purpose, at first the characterization of Cobalt-Zinc Ferrite Magnetic Nanoparticles (CZF-MNPs) coated with biocompatible Dimercaptosuccinic Acid (DMSA) was investigated using of Transmission Electron Microscopy (TEM), Fourier Transform Infrared (FTIR) and Atomic Absorption Spectrophotometer (AAS). In following, the cytotoxicity effects of CZF-MNPs and CZF-MNPs @ DMSA were investigated on human prostate cancer cell lines, HPCs, (PC3 and DU145). The results showed that the average size of bare and coated nanoparticles was about 16 and $40 \mathrm{~nm}$. The FTIR spectra results showed the presence of DMSA cover on the surface of nanoparticles. Furthermore, in vitro MTT assay CZF-MNPs @ DMSA at high concentrations (1.2 and $1.5 \mathrm{mM} \mathrm{Fe}$ ) study results showed that they have some cytotoxicity on HPCs (PC3 and DU145).
\end{abstract}

Keywords: Cobalt-zinc ferrite; DMSA coated; Cytotoxicity; Human prostate cancer cells (HPCs); MTT assay

\section{Introduction}

The applicability of nanotechnology is strongly being attracted for various applications such as cytotoxicity, magnetic resonance imaging, biomedical applications, drug delivery and cancer therapy [1-6]. Recently, Spinel ferrite nanoparticles with different coatings have been widely investigated, due to their desirable magnetic properties in biomedicine and bioengineering fields [7-9]. These magnetic nanoparticles should have high magnetization values and size smaller than 100 nanometers. Also, these materials should have low toxicity and high biocompatible $[10,11]$.

Various types of monomers such as DMSA were evaluated as anchors for simple attachment of polymer coatings on magnetic nanoparticles [12].

Toxicity of nanoparticles on the human health is one main feature for successful application of nanoparticles in medicine. Recently, the cytotoxicity effects of DMSA- $\mathrm{Fe}_{2} \mathrm{O}_{3}$ by tetrazolium dye assay on human aortic endothelial cells were reported and were shown that DMSA$\mathrm{Fe}_{2} \mathrm{O}_{3}$ has some cytotoxicity [2]. On the other, choosing an appropriate surface coating for the desired application is important and complex [13]. There is controversy about cell viability in this regard. For instance, Pisanic et al. have demonstrated that DMSA coated nanoparticles were toxic to neurons [14], but in another study Wilhelm et al. have shown that DMSA coated nanoparticles were non-toxic to HeLa cells [15].

In this paper, the characterization of magnetic nanoparticles of $\mathrm{Co}_{0.5} \mathrm{Zn}_{0.5} \mathrm{Fe}_{2} \mathrm{O}_{4}$ and $\mathrm{Co}_{0.5} \mathrm{Zn}_{0.5} \mathrm{Fe}_{2} \mathrm{O}_{4} @$ DMSA were investigated by Transmission Electron Microscopy (TEM), Fourier Transform Infrared Spectroscopy (FTIR) and Atomic Absorption Spectrophotometer (AAS). Also, the cytotoxicity effects of CZF-MNPs and CZF-MNPs @ DMSA were investigated for the first time on human prostate cancer cell lines, HPCs, (PC3 and DU145).

\section{Experimental}

\section{Preparation and characterization of MNPs}

Synthesis of cobalt-zinc ferrite nanoparticles and its coated with DMSA described by author and co-worker, previously [16]. Nanoparticles size and morphology were investigated by Transmission Electron Microscopy (EM 900 model, Co Zeiss.). The FTIR of nanoparticles were evidenced with FT Infrared Spectroscope (JASCO, FT/IR-6300, Japan) in the range of $400-4000 \mathrm{~cm}^{-1}$. The content of $\mathrm{Fe}^{2+}$ in $\mathrm{Co}_{0.5} \mathrm{Zn}_{0.5} \mathrm{Fe}_{2} \mathrm{O}_{4}$ and $\mathrm{Co}_{0.5} \mathrm{Zn}_{0.5} \mathrm{Fe}_{2} \mathrm{O}_{4} @$ DMSA MNPs was done by atomic absorption spectrophotometer (Shimadzu, AA-680).

\section{Cell culture}

HPCs (DU145 and PC3) were purchased from the National Cell Bank of Iran, Pasteure Institute of Iran. They were cultured in Roswell Park Memorial Institute medium (RPMI) media supplemented with 1\% antibiotics (100 units/ml penicillin and $100 \mu \mathrm{g} / \mathrm{ml}$ streptomycin) and $10 \%$ fetal bovine serum until the third passage before experiments were performed. All the cell culture materials were from Gibco, USA. Cells were grown to confluence at $37^{\circ} \mathrm{C}$ in $5 \% \mathrm{CO}_{2} /$ air.

\section{In vitro cytotoxicity (MTT assay)}

The cytotoxicity of coated and uncoated magnetic nanoparticles

*Corresponding author: Daryoush Shahbazi-Gahrouei, Department of Medical Physics and Medical Engineering, Isfahan University of Medical Sciences, Isfahan, Iran, Tel: +98-311-7922495; E-mail: shahbazi24@yahoo.com, shahbazi@med.mui.ac.ir

Received October 22, 2013; Accepted October 26, 2013; Published October 28 2013

Citation: Shahbazi-Gahrouei D, Ghasemian Z, Abdolahi M, Manouchehri S Javanmard SH, et al. (2013) In vitro Evaluation of Cobalt-Zinc Ferrite Nanoparticles Coated with DMSA on Human Prostate Cancer Cells. J Mol Biomark Diagn 4: 154. doi:10.4172/2155-9929.1000154

Copyright: (c) 2013 Shahbazi-Gahrouei D, et al. This is an open-access article distributed under the terms of the Creative Commons Attribution License, which permits unrestricted use, distribution, and reproduction in any medium, provided the original author and source are credited 
was evaluated using standard 3-(4, 5-dimethylthiazol-2yl)-2,5diphenyltetrazolium bromide (MTT) based colorimetric assay. Cancer cells were cultured in 96 well plates in $200 \mu \mathrm{L}$ media per well at a density of $10^{4}$ cells/well. After $24 \mathrm{~h}$ of incubation, the media was replaced with fresh media containing of different concentration $(0.3,0.6,0.9,1.2$ and $1.5 \mathrm{~m} \mathrm{~mol}$ ) of coated and uncoated MNPs, and incubated for 24 h. After which time, the plate supernatant was discarded and $100 \mathrm{ml}$ solution of MTT (25 mg MTT in $5 \mathrm{ml}$ colorless RPMI solution) was added into each well. After $3 \mathrm{~h}$ of incubation with MTT, each well of the plate was added $100 \mathrm{ml}$ Dimethyl Sulfoxide (DMSO) to dissolve the purple formazan. In order to being deposited the remaining nanoparticles in the solution of the wells, 96 well plate was centrifuged at $2500 \mathrm{rpm}$ for $10 \mathrm{~min}$. After centrifuge, supernatant was transferred to another 96 -well plate. Then; absorbance of each well was read by using
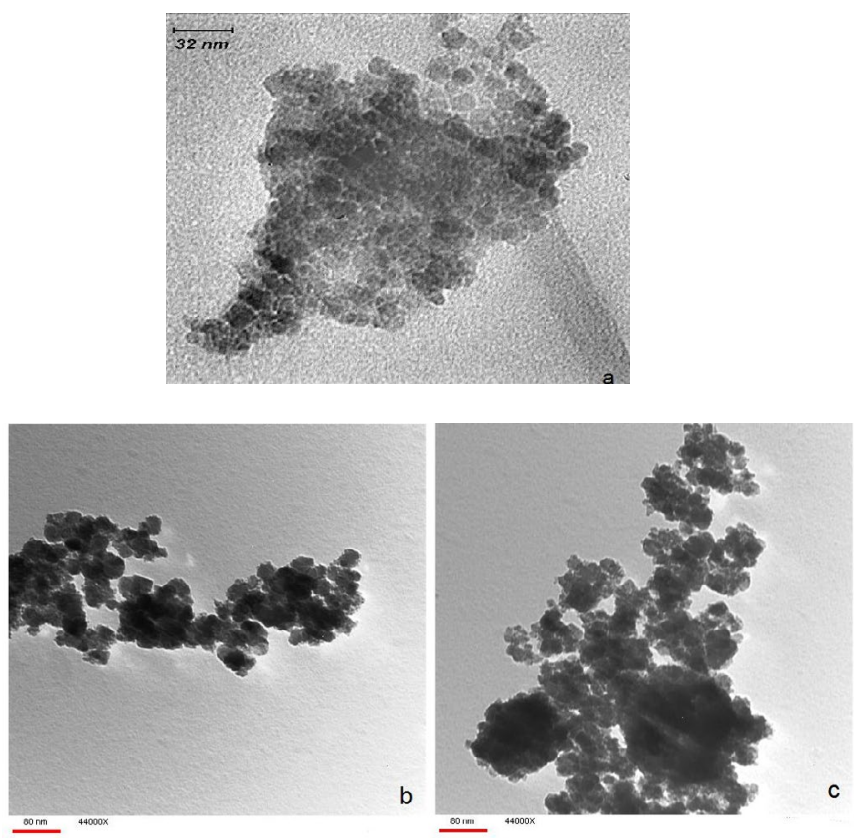

Figure 1: TEM bright field images of a) CZF-MNPs (co-precipitation) and b) and c) two TEM bright field images of CZF-MNPs @ DMSA (co-precipitation).

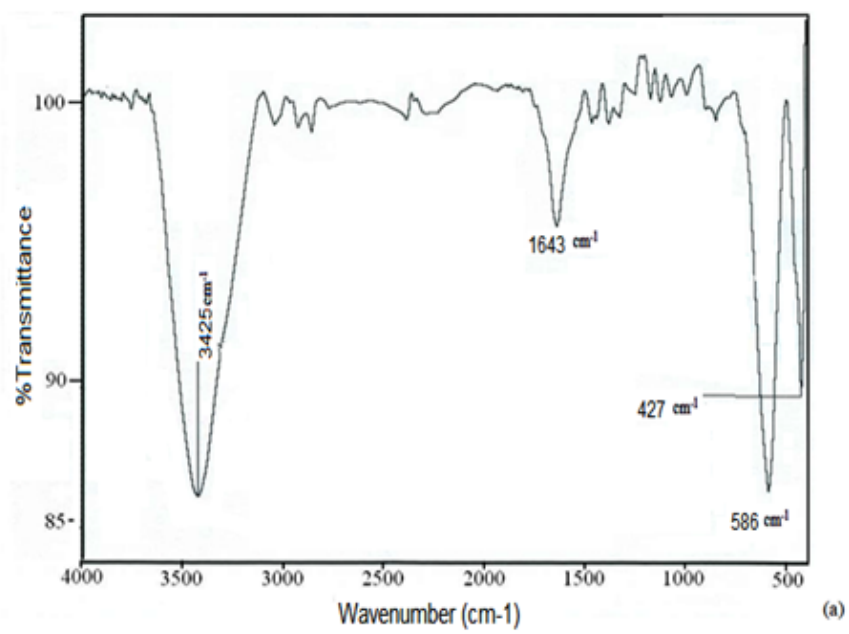

Figure 2a: FTIR spectra of CZF-MNPs. an Enzyme-Linked Immunosorbent Assay (ELISA) plate reader with a reference wavelength of $570 \mathrm{~nm}$. The cell viability was determined by the following formula:

$$
\% \text { Cell viability }=\frac{\text { Mean absorbance in test wells }}{\text { Mean absorbance in control wells }} \times 100
$$

All experiments were repeated three times. All control groups included control samples containing PC 3 and DU145 and RPMI 1640 culture medium in the absence of nanoparticles.

\section{Incubation of cells with the bare and coated MNPs}

For the preparation of gelatin suspension $1 \mathrm{~g}$ of powder gelatin dissolved in $50 \mathrm{ml}$ of PBS solution, and put it for $30 \mathrm{~min}$ in water bath to obtain uniform solution. Two prostate cell lines (PC3 and DU145) incubated with different concentrations $(0.5,1$ and $1.5 \mathrm{~m} \mathrm{~mol})$ of CZF-MNPs and CZF-MNPs@DMSA for $2 \mathrm{~h}$ at room temperature in RPMI 1640 culture medium. In order to separation of particles sticking together, before adding bare and coated MNPs suspension with different concentrations into RPMI culture medium were sonicated for $20 \mathrm{~min}$. Then, the cells were washed three times with PBS solution. In the next step, $1 \mathrm{~mL}$ of gelatin suspension is added to $1.5 \mathrm{~mL}$ Eppendorf tubes with different concentrations, and stirring to obtain uniform solution. Then, the Eppendorf tubes with different concentrations are placed on ice powder, until obtained solution is solidity. Control groups are containing only $1 \mathrm{ml}$ of gelatin suspension.

\section{In vitro $\mathrm{MR}$ imaging}

These Eppendorf tubes with different concentrations were used to experiment the in vitro MRI characterizations. T2-weighted images were obtained using a 1.5 T MRI scanner (1.5 T GE Medical system).

\section{Results and Discussion}

\section{Characterizations}

The surface morphology was evaluated by Transmission Electron Microscopy (TEM) (EM 900 model, Co Zeiss, operating at $80 \mathrm{keV}$ ). TEM bright field images of CZF-MNPs and CZF-MNPs @ DMSA (coprecipitation) are shown in Figure 1. This figure shown that particles have almost spherical structures and also the average particles size of CZF-MNPs and CZF-MNPs @ DMSA (co-precipitation) are 16 and 40 $\mathrm{nm}$, respectively.

In order to prove the presence of the surface coated, the MNPs pre- and post- DMSA coated were evaluated using the FTIR method (Figure 2). Peaks at 3425 and $1643 \mathrm{~cm}^{-1}$ are related to $\mathrm{OH}$ bands which indicated the presence of water in the structure of matter. Peaks at 580 and $427 \mathrm{~cm}^{-1}$ indicated that the spinel structure was formed. Peaks at 928, 1160, 1360 and $1701 \mathrm{~cm}^{-1}$ which showed in Figure (2b) indicated that DMSA coated on the CZF-MNPs surface. The FTIR diagrams analysis confirmed the formation of Co-Zn ferrite phase and showed the presence of cover on the surface of nanoparticles and also the presence of water content in the samples. The concentration of $\mathrm{Fe}$ in CZF-MNPs and CZF-MNPs @ DMSA are 325 and 225 ppm. This loss concentration indicated the presence of DMSA coated on the surface of the bare MNPs.

\section{In vitro $\mathrm{MR}$ imaging}

The ability and suitability of synthesized nanoparticles as a MR contrast agents, was also confirmed with MR imaging techniques. T2-weighted images were obtained with a (1.5 T GE Medical system) 


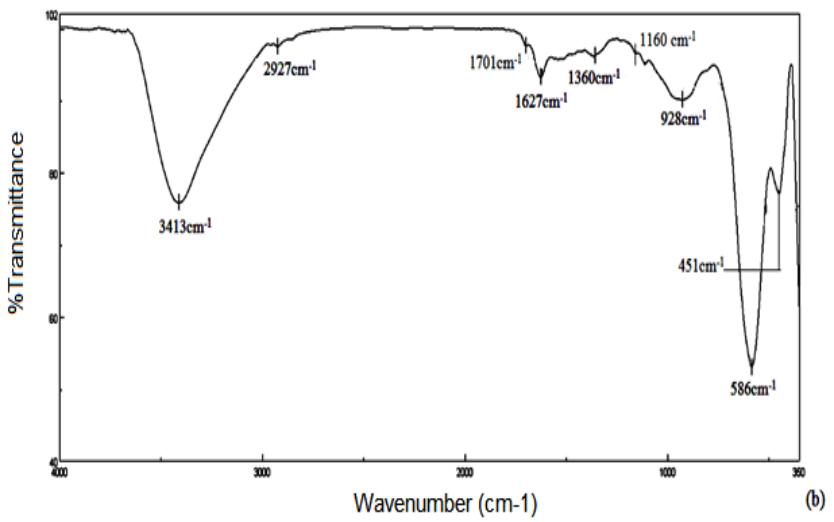

Figure 2b: FTIR spectra of CZF-MNPs @ DMSA.

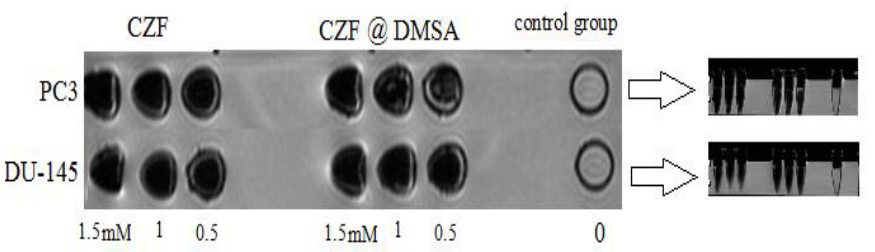

Figure 3: $T_{2}$-weighted magnetic resonance images at various Fe concentrations CZF-MNPs and CZF-MNPs@ DMSA (1.5 T, Fast spin echo sequence: $\mathrm{T}_{\mathrm{R}}=2520$ $\mathrm{ms}, \mathrm{T}_{\mathrm{E}}=102 \mathrm{~ms}$, room temperature) The $\mathrm{T}_{2}$-weighted MR image shows that CZF-MNPs and CZF-MNPs@ DMSA induce a negative contrast. In addition, for the same Fe content, the enhancement in contrast using CZF-MNPs is the same as the amount of CZF-MNPs @ DMSA.

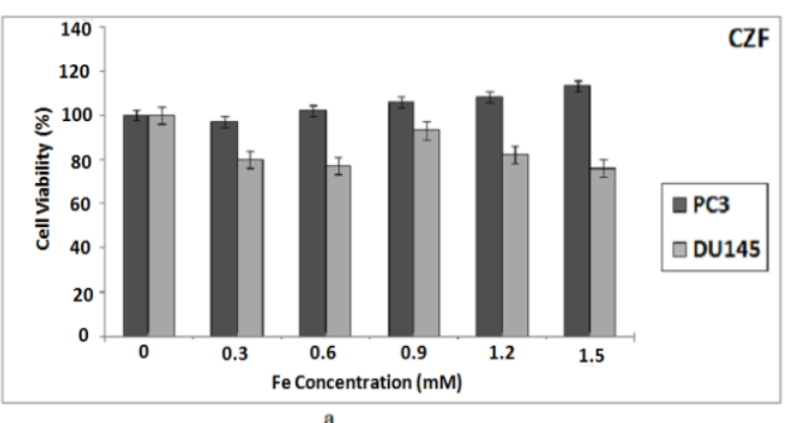

Figure 4a: The cytotoxicity effect of CZF-MNPs and CZF-MNPs @ DMSA.

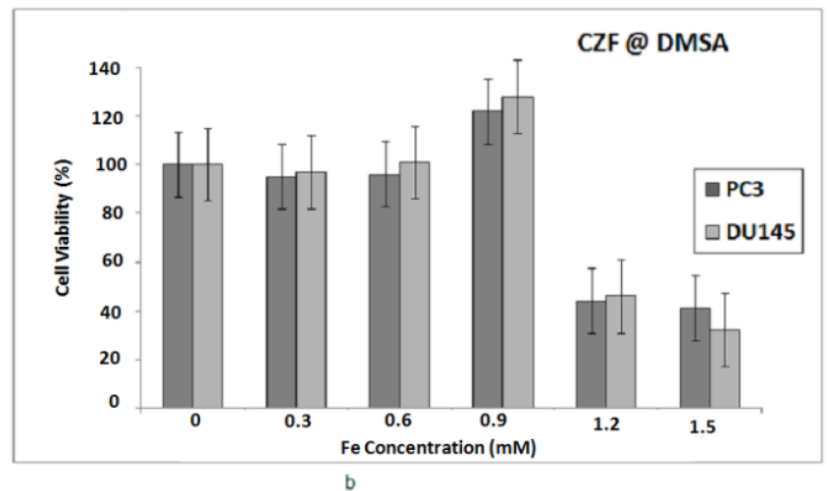

Figure 4b: The cytotoxicity effect of CZF-MNPs in two HPCs (PC3 and DU145) Data as mean $\pm \operatorname{SD}(n=6)$. scanner. The results displayed in Figure 3 demonstrate that both uncoated and coated nanoparticles have been imported in PC3 and DU145 cell lines and induced grate signal intensity reduction.

\section{In vitro cytotoxicity (MTT assay)}

In vitro cell viability of PC3 and Du145 of HPCs after exposure to CZF-MNPs and CZF-MNPs @ DMSA was assessed by MTT assay at different concentrations of Fe. The viability of PC3 cells do not decreased versus control group by increasing Fe concentrations of CZFMNPs, but only at a concentration of $0.3 \mathrm{mM}$ has negligible toxicity (3\%). The overall, the CZF-MNPs indicated that a high viability against PC 3 cells. The viability of Du 145 cells at different concentrations of Fe of CZF-MNPs is more than $75 \%$ (Figure 4a).

As shown in Figure 4b, the same viability of PC3 and Du145 HPCs after exposure to CZF-MNPs @ DMSA was evaluated by MTT assay at different concentrations of $\mathrm{Fe}$.

The viability of PC3 and Du145 cells is more than 75\%, but only at concentrations of 1.2 and $1.5 \mathrm{mM}$ it is less than $50 \%$. The toxicity of coated nanoparticles at high concentrations ( 1.2 and $1.5 \mathrm{mM}$ of $\mathrm{Fe}$ ) is more than that of pure nanoparticles.

In this study CZF-MNPs @ DMSA at concentration of $0.9 \mathrm{mM} \mathrm{Fe}$ (after $24 \mathrm{~h}$ incubation), leading to proliferation of PC-3 and Du145 cancer cells, this result is similar to study which done by Ge et al. They have shown that after $24 \mathrm{~h}$ incubation of $\mathrm{Fe}_{2} \mathrm{O}_{3} @$ DMSA with human aortic endothelial cells at concentration of $0.01 \mathrm{mg} / \mathrm{ml}$, leading to proliferation of human aortic endothelial cells. In addition, at low concentration of 0.001 and $0.02 \mathrm{mg} / \mathrm{ml}$, viability of human aortic endothelial cells is equal to control group (100\%). The same result was obtained at 0.3 and $0.6 \mathrm{mM}$ concentration of Fe (viability of PC 3 and DU145 cancer cell lines is approximately $100 \%$ ). Thus, certain doses of nanoparticles coated with DMSA can be used for biological researches, and in these special doses, no side effect was observed on healthy tissues.

\section{Conclusions}

The viability of the HPCs cells is dependent to concentration of MNPs. Also, the toxicity of these nanoparticles should be studied on the other cancer cell lines. The results showed that $14 \mathrm{~nm}$ CZFMNPs did not induce toxicity in PC3 HPCs. Rather, increasing concentrations of CZF-MNPs is leading to proliferation of this cell line. It is also surprising, both in this study and another study which done by Ge et al. in 2013 [2], nanoparticles coated with DMSA, in a certain concentration, not only are not toxic but also are leading to proliferation of cancer cells. The mechanisms relative to proliferation of cancer cells are unknown. Therefore, further investigation is needed to demonstrate the cell viability effect. As a suggestion, the cytotoxicity effect of magnetic nanoparticles is needed, not only on cancer cell lines but also on normal cell lines.

\section{References}

1. Yallapu MM, Othman SF, Curtis ET, Gupta BK, Jaggi M, et al. (2011) Multifunctional magnetic nanoparticles for magnetic resonance imaging and cancer therapy. Biomaterials 32: 1890-1905.

2. Ge G, Wu H, Xiong F, Zhang Y, Guo Z, et al. (2013) The cytotoxicity evaluation of magnetic iron oxide nanoparticles on human aortic endothelial cells. Nanoscale Research Letters 8: 1-10.

3. Gryparis EC, Hatziapostolou M, Papadimitriou E, Avgoustakis K (2007) Anticancer activity of cisplatin-loaded PLGA-mPEG nanoparticles on LNCaP prostate cancer cells. Eur J Pharm Biopharm 67: 1-8. 
Citation: Shahbazi-Gahrouei D, Ghasemian Z, Abdolahi M, Manouchehri S, Javanmard SH, et al. (2013) In vitro Evaluation of Cobalt-Zinc Ferrite Nanoparticles Coated with DMSA on Human Prostate Cancer Cells. J Mol Biomark Diagn 4: 154. doi:10.4172/2155-9929.1000154

Page 4 of 4

4. Debouttière PJ, Roux S, Vocanson F, Billotey C, Beuf O, et al. (2006) Design of Gold Nanoparticles for Magnetic Resonance Imaging. Adv Funct Mater 16: 2330-2339.

5. Horák D, Babic M, Jendelová P, Herynek V, Trchová M, et al. (2009) Journal of Magnetism and Magnetic Materials 321: 1539.

6. Jain TK, Richey J, Strand M, Leslie-Pelecky DL, Flask CA, et al. (2008) Magnetic nanoparticles with dual functional properties: drug delivery and magnetic resonance imaging. Biomaterials 29: 4012-4021.

7. Doulabi FM, Mohsen-Nia M (2013) Magnetic cobalt-zinc ferrite/PVAc nanocomposite: synthesis and characterization. Iranian Polymer Journal 22: 9-14.

8. Hoque SM, Srivastava C, Srivastava N, Venkateshan N, Chattopadhyay K (2013) Journal of Materials Science 48: 812

9. Girgis E, Wahsh MM, Othman AG, Bandhu L, Rao K (2011) Synthesis, magnetic and optical properties of core/shell Co1-xZnxFe2O4/SiO2 nanoparticles. Nanoscale Res Lett 6: 460 .
10. Cullity BD, Stock SR (2001) Elements of X-ray Diffraction; Prentice hall Upper Saddle River, NJ.

11. Muroi M, Street R, McCormick P, Amighian (2001) J. Physical Review B 63 : 184414

12. Fauconnier N, Pons JN, Roger J, Bee A (1997) Thiolation of Maghemite Nanoparticles by Dimercaptosuccinic Acid J Colloid Interface Sci 194: 427-433.

13. Latorre M, Rinaldi C (2009) Applications of magnetic nanoparticles in medicine: magnetic fluid hyperthermia. P R Health Sci J 28: 227-238.

14. Pisanic TR 2nd, Blackwell JD, Shubayev VI, Fiñones RR, Jin S (2007) Nanotoxicity of iron oxide nanoparticle internalization in growing neurons. Biomaterials 28: 2572-2581.

15. Wilhelm C, Billotey C, Roger J, Pons JN, Bacri JC, et al. (2003) Intracellular uptake of anionic superparamagnetic nanoparticles as a function of their surface coating. Biomaterials 24: 1001-1011.

16. Manouchehri S Ghasemian Z, Shahbazi-Gahrouei D, Abdolahi M (2013) ChemXpress 147 